\title{
Una secuencia didáctica para el aprendizaje de los conceptos de adición, sustracción y multiplicación de polinomios en estudiantes con limitación auditiva ${ }^{1}$
}

\author{
A teaching sequence for learning the concepts of addition, subtraction \\ and multiplication of polynomials in students with limited hearing
}

Uma seqüência de ensino para a aprendizagem dos conceitos de adição, subtração e multiplicação de polinômios em estudantes com audição limitada

Recibido: mayo de 2013

Aceptado: agosto de 2013
Sandra Lucía Romero Pulido ${ }^{2}$

Liliana Patricia Ospina Marulanda ${ }^{3}$

\section{Resumen}

Este proyecto de investigación tiene como propósito lograr la comprensión de los conceptos de adición, sustracción y multiplicación de polinomios en estudiantes con limitación auditiva de la Institución Educativa CASD Hermógenes Maza de la ciudad de Armenia a través situaciones didácticas, que privilegien el uso de recursos manipulativos y diferentes formas de representación, para ello se utilizará la metodología de ingeniería didáctica, mediante la cual se espera analizar el nivel de comprensión alcanzado por los estudiantes en la construcción de los conceptos y el papel que juegan las diferentes formas de representación, el uso de recursos manipulativos y los elementos propios del álgebra geométrica como facilitadores del aprendizaje en los estudiantes con limitación auditiva.

Palabras clave: Secuencia didáctica; limitación auditiva; necesidades educativas especiales; matemáticas escolares; álgebra; polinomios.

\begin{abstract}
This research project aims to develop an understanding of the concepts of addition, subtraction and multiplication of polynomials in students with limited hearing of School CASD Maza Hermogenes Armenia City through didactic situations that favor the use of manipulatives resources and different forms of representation, this will use the didactic engineering methodology by which it is expected to analyze the level of understanding achieved by students in the construction of concepts and the role of different forms of representation, the use of manipulatives and resources elements of the geometric algebra as facilitators of learning in students with limited hearing.
\end{abstract}

1 Artículo de Investigación

2 Universidad del Quindío. Colombia. Contacto: sandra33816@hotmail.com

3 Universidad del Quindío. Colombia. Contacto: lpospina@uniquindio.edu.co 
Keywords: Teaching sequence; limited hearing, special educational needs school math, algebra, polynomials.

\section{Resumo}

Este projeto de pesquisa tem como objetivo desenvolver a compreensão dos conceitos de adição, subtração e multiplicação de polinômios em estudantes com audição limitada de Escola CASD Maza Hermogenes Armenia Cidade através de situações didáticas que favoreçam o uso de recursos manipulativos e diferentes formas de representação, isso vai usar a metodologia da engenharia didática pela qual espera-se analisar o nível de compreensão alcançado pelos alunos na construção de conceitos eo papel das diferentes formas de representação, o uso de manipuláveis e elementos de recursos da álgebra geométrica como facilitadores da aprendizagem nos alunos com audição limitado.

Palavras-chave: seqüência de ensino; audiência limitada, matemática necessidades especiais de educação escolar, álgebra, polinômios.

\section{Presentación del problema}

En las políticas estatales del MEN (2005), se han definido lineamientos para la atención educativa a poblaciones vulnerables, identificadas como aquellas que por sus diferencias socioculturales económicas y/o biológicas han permanecido fuera del sistema educativo. Pero no se trata únicamente de abrir las aulas regulares a dichas poblaciones, ya que hay otros aspectos que reflexionar, por ejemplo: ¿Cómo les va a los estudiantes con discapacidad que se incluyen en las aulas?, ¿Cuáles son los índices de deserción escolar de dichas poblaciones al no encontrar singularidades pedagógicas en las instituciones comunes?, ¿Cuáles son los niveles de desempeño académico de los estudiantes con discapacidad?, ¿Cuáles son las estrategias didácticas apropiadas para los procesos de enseñanza-aprendizaje?. Esta realidad exige del maestro una nueva mirada de apertura frente a la diversidad de los estudiantes que llegan a las aulas, entre ellos los de necesidades educativas especiales. En la investigación se trabajará con estudiantes de grado $11^{\circ}$ con limitación auditiva de la institución educativa CASD de Armenia, quienes presentan dificultades en el área de matemáticas, lo que se evidencia en su rendimiento académico y en los resultados de las pruebas SABER $11^{\circ}$, a los estudiantes se les dificulta la comprensión de conceptos básicos de cálculo, debido a la falta de estabilidad de conceptos previos, como son los relacionados con el álgebra.
La presente propuesta de investigación tiene como propósito lograr en los estudiantes la comprensión de los conceptos de adición, sustracción y multiplicación de polinomios a través de situaciones didácticas, que privilegien el uso de recursos manipulativos y diferentes formas de representación.

\section{Marco de referencia conceptual}

En el marco de referencia conceptual se plantean conceptos teóricos básicos que fundamentan ésta propuesta de investigación, la principal fuente es la teoría de situaciones didácticas, en el que se involucran otros elementos teóricos, entre los cuales están: el aprendizaje del álgebra, y el aprendizaje de las matemáticas en personas con deficiencia auditiva.

Teoría de Situaciones Didácticas. Esta teoría se sustenta en una concepción constructivista del aprendizaje, en palabras de Brousseau (1986), citado por D'amore (2006: 243): "El estudiante aprende adaptándose a un ambiente que es factor de contradicciones, de dificultades, de desequilibrios, un poco como lo hace la sociedad humana. Este saber, fruto de la adaptación del alumno, se manifiesta por respuestas nuevas que son la prueba del aprendizaje (...)".

Teniendo en cuenta que el aprendizaje de los conceptos es el resultado de un proceso mediado 
por diversas situaciones, se considera la teoría de situaciones didácticas, como un elemento de saber importante que permite comprender las acciones de docentes y estudiantes, para al mismo tiempo planear y producir elementos didácticos adaptados a sus necesidades. Al interior de ella se consideran algunos conceptos fundamentales: situaciones didácticas de acción, formulación, validación e institucionalización; así como situaciones a-didácticas, variables didácticas, devolución, transposición didáctica y el contrato didáctico.

El aprendizaje del álgebra. Para kieran (1989: 229), aprender álgebra no es meramente hacer explícito lo que estaba implícito en la aritmética. El álgebra requiere un cambio en el pensamiento del estudiante de las situaciones numéricas concretas a proposiciones más generales sobre números y operaciones.

Pensamiento algebraico. El MEN (2006: 67) en los estándares básicos de competencias, define el pensamiento variacional y los sistemas algebraicos y analíticos, como el tipo de pensamiento que tiene que ver con el reconocimiento, la percepción, la identificación y la caracterización de la variación y el cambio en los diferentes contextos, así como su descripción, modelación y representación en distintos sistemas o registros simbólicos, ya sean verbales, icónicos, gráficos o algebraicos.

Dificultades y errores en el aprendizaje del álgebra. En el proceso de transición desde un modo informal de representación a uno formal, que es el que se adquiere con el manejo del álgebra, los estudiantes suelen cometer algunos errores, al respecto, Palarea y Socas (1999: 10), han estudiado los obstáculos que frenan el progreso del conocimiento del alumno y que son inherentes al aprendizaje de conceptos y procedimientos en el inicio del acercamiento al álgebra, las dificultades pueden abordarse desde varias perspectivas: asociadas a la complejidad de los objetos del álgebra, asociadas a los procesos de pensamiento en álgebra, asociadas a los procesos de enseñanza desarrollados para el aprendizaje del álgebra y asociadas a los procesos de desarrollo cognitivo de los alumnos.
Por la complejidad que representa el aprendizaje del álgebra, desde el punto de vista epistemológico, la didáctica del álgebra debe ser revisada para poder implementar estrategias que permitan una construcción significativa del aprendizaje, en el caso de la presente propuesta, se hace necesario dar una mirada teórica a las características que deben tener los procesos de enseñanza-aprendizaje para los estudiantes sordos.

El MEN (2006:5) dio algunas orientaciones pedagógicas para la atención educativa a estudiantes con limitación auditiva, las cuales son importantes en el momento de planear situaciones didácticas de tal forma que respondan a sus necesidades y posibiliten la evolución de los conceptos.

Necesidades Educativas Especiales (NEE). Se definen como estudiantes con Necesidades Educativas Especiales a aquellas personas con capacidades excepcionales, o con alguna discapacidad de orden sensorial, neurológico, cognitivo, comunicativo, psicológico o físico-motriz, y que puede expresarse en diferentes etapas del aprendizaje. MEN (2009).

Implicación e importancia de los recursos visuales en la construcción de conceptos matemáticos para las personas sordas. La percepción visual es una herramienta importante para la enseñanza de las matemáticas en los estudiantes sordos, para Márquez (2011:34) el contexto donde se desenvuelven los problemas debe privilegiar la presencia de condiciones visualmente significativas en los materiales, recursos y representaciones. Esta indicación se traduce en la incorporación de recursos didácticos que ofrezcan información visual necesaria para la representación y tratamiento de los objetos de aprendizaje en el contexto del problema. Con lo anterior se hace referencia a la importancia que tiene la visualización en los procesos de enseñanza-aprendizaje de los estudiantes sordos, otros autores como Larrubia y Gonzáles (2006:8) han demostrado que actividades que comprometen en mayor medida la visualización como son las gráficas y las representaciones no verbales, favorecen la comprensión y la representación mental del alumnado sordo y son el mejor vehículo para la adquisición del conocimiento en estos estudiantes 


\section{Conclusiones}

Se espera identificar las necesidades de carácter didáctico que tienen los estudiantes sordos para el aprendizaje del concepto de adición, sustracción y multiplicación de polinomios, así mismo validar secuencias didácticas que permitan facilitar la comprensión del concepto de adición, sustracción y multiplicación de polinomios en estudiantes con limitación auditiva, evaluar el papel que juegan el uso de recursos manipulativos, visuales y de diferentes formas de representación, en el aprendizaje de los conceptos mencionados en los estudiantes.

\section{Referencias}

Artigue, M; Douady, R; Moreno, L; Gómez, P. (1995). Ingeniería Didáctica en Educación Matemática. Un esquema para la investigación y la innovación en la enseñanza y el aprendizaje de las matemáticas. Bogotá: Grupo Editorial Iberoamericana. Recuperado en http://funes.uniandes.edu. co/676/1/Artigueetal195.pdf

D`Amore, B. (2006). Didáctica de la matemática. Bogotá: Magisterio.
Hernández, J; Muñoz, M; Paralea, M; Ruano, R; et-al (2008). Materiales manipulativos para la enseñanza y el aprendizaje del álgebra en la educación obligatoria. Universidad de la Laguna, España. Recuperado en http://es.scribd.com/doc/128579058/ Investigacion-Cientifica-en-El-Peru

Kieran, C \& Filloy Yague (1989). "El aprendizaje del álgebra escolar desde una perspectiva psicológica”. Investigación y experiencias didácticas. University of London, Institute of Education, Inglaterra. Traducción de Luis Puig. Recuperado en http://ddd.uab.es/pub/ edlc/02124521v7n3p229.pdf

Larrubia, J; González, J. (2006). Aprendizaje matemático en alumnado sordo integrado en aulas ordinarias de E.S.O y bachillerato. Departamento de didáctica de las matemáticas en la Universidad de Málaga, España. Recuperado en http://www.gonzalezmari.es/ atenci_n_diversidad_sordos_oyentes_aula_ de_matem_ticas.pdf

Márquez, Héctor A. (2011). Orientaciones para el diseño de situaciones didácticas en matemáticas a estudiantes sordos. Bogotá: Ministerio de Educación Nacional-INSOR 